Como citar este artículo: López MP, Guete CN, Ospina J. Detección de cepas de Klebsiella pneumoniae productoras de betalactamasas de espectro extendido aisladas en un centro clínico de alta complejidad en Santa Marta, Colombia. Revista Biosalud 2015; 14(2): 63-70. DOI: 10.17151/biosa.2015.14.2.6

\title{
DETECCIÓN DE CEPAS DE Klebsiella pneumoniae PRODUCTORAS DE BETALACTAMASAS DE ESPECTRO EXTENDIDO AISLADAS EN UN CENTRO CLÍNICO DE ALTA COMPLEJIDAD EN SANTA MARTA, COLOMBIA
}

\author{
Mónica Patricia López ${ }^{1}$ \\ Cindi Natali Guete ${ }^{2}$ \\ Jacqueline Ospina ${ }^{3}$
}

\begin{abstract}
RESUMEN
Introducción: Klebsiella pneumoniae es uno de los principales microorganismos productores de betalactamasas de espectro extendido (BLEE) comúnmente aislados en centros clínicos, convirtiéndose en el principal responsable de enfermedades infecciosas oportunistas. Materiales y Métodos: Mediante el sistema API 10S se identificaron cepas de K. pneumoniae provenientes de cualquier tipo de muestras biológicas. La detección de las BLEE se efectuó según las recomendaciones de Clinical and Laboratory Standards Institute. Resultados: Entre los meses de septiembre de 2014 y marzo de 2015, 18 cepas de K. pneumoniae fueron productoras de BLEE y exhibiendo un patrón fenotípico caracterizado por la resistencia a las aminopenicilinas, ureidopenicilinas, carboxipenicilinas y cefalosporinas de primera, segunda y tercera generación. Presentaron también resistencia a las combinaciones con inhibidores de bectalactamasas, así como a algunos aminoglucósidos tales como tobramicina, gentamicina y netilmicina. Manteniendo la sensibilidad a la amikacina, trimetoprim/sulfametoxazol, quinolonas y carbapenems. Discusión: La prevalencia de las cepas de K. pneumoniae productoras de BLEE
\end{abstract}

fue similar a la reportada en otras ciudades de Colombia. Difiriendo en la resistencia exhibida frente a los inhibidores de betalactamasas y carbapenems, aunque en bajos porcentajes para estos últimos. Resaltando una alta sensibilidad a la amikacina.

Palabras clave: betalactamasas, Klebsiella pneumoniae, productoras, resistencia.

\section{DETECTION OF Klebsiella pneumoniae STRAINS PRODUCERS OF EXTENDED SPECTRUM BETA-LACTAMASES ISOLATED IN A HIGH COMPLEXITY CLINICAL CENTER IN SANTA MARTA, COLOMBIA}

\begin{abstract}
Introduction: Klebsiella pneumoniae is one of the main microorganisms producing extended spectrum beta-lactamase (ESBL) commonly isolated in clinical centers, becoming the main responsible for opportunistic infectious diseases. Materials and Methods: Strains of $K$. pneumoniae from any type of biological samples were identified using the API 10E system. ESBLs
\end{abstract}

\footnotetext{
${ }^{1}$ Estudiante de Biología. Facultad de Ciencias Básicas, Universidad del Magdalena. Santa Marta, Colombia. Correo electrónico: monica.3003@hotmail.com

${ }^{2}$ Estudiante de Biología. Facultad de Ciencias Básicas, Universidad del Magdalena. Santa Marta, Colombia. Autora para correspondencia. Correo electrónico: cindyguettes@gmail.com

${ }_{3}^{3}$ Bacterióloga. Laboratorio de Microbiología, Clínica Mar Caribe. Santa Marta, Colombia. Correo electrónico: jospinaa@ hotmail.com
} 
detection was performed as recommended by the Clinical and Laboratory Standards Institute. Results: Between September 2014 and March2015, 18 strains of K. pneumoniae produced ESBL and exhibited a phenotypic pattern characterized by resistance to aminopenicillins ureidopenicillins, carboxypenicillins and cephalosporins of first, second and third generation. They also showed resistance to combinations with bectalactamases inhibitors as well as to some aminoglycosides such as tobramycin, gentamicin and netilmicin, maintaining sensitivity to

\section{INTRODUCCIÓN}

Son varios los mecanismos de resistencia que pueden exhibir las enterobacterias ante los diferentes tipos de antimicrobianos, entre ellos, variaciones de la permeabilidad, alteraciones de los sitios diana y la producción de enzimas inactivantes, siendo este último el principal responsable de la resistencia ante diferentes antibióticos (1). Dentro de tales enzimas se encuentran las betalactamasas, que son producidas tanto por bacterias Gram-positivas como Gram-negativas confiriéndole resistencia contra los antibióticos betalactámicos $(2,3)$.

Dentro de los microorganismos comúnmente aislados en centros clínicos se encuentra Klebsiella pneumoniae que, junto a Escherichia coli, es uno de los principales productores de betalactamasas de espectro extendido (BLEE) y responsable de un gran número de infecciones nosocomiales (4).

Klebsiella, al igual que gran parte de las enterobacteriáceas, muestra un patrón de resistencia natural mediado por betalactamasas cromosómicas, presentando así resistencia in vitro a las aminopenicilinas y carboxipenicilinas, sensibilidad disminuida a las ureidopenicilinas, conservando la sensibilidad frente a cefalosporinas, cefamicinas, monobactámicos, carbapenémicos y asociaciones con inhibidores de betalactamasas $(1,5)$. amikacin, trimethoprim/sulfamethoxazole, quinolones and carbapenems. Discussion: The prevalence of $K$. pneumoniae strains producing ESBL was similar to that reported in other cities of Colombia, differing in the resistance exhibited against beta-lactamase and carbapenems inhibitors, although in lower percentages for the latter, and emphasizing high sensitivity to amikacin.

Key words: ß-lactamases, Klebsiella pneumoniae, producers, resistance.

Son varias las clasificaciones que se le han dado a las betalactamasas debido a su gran cantidad y heterogeneidad, siendo las de Ambler y BushJacoby-Medeiros las más usadas en la actualidad (6).

Específicamente, las BLEE se encuentran dentro del grupo 2be (7) -clase "A" de Ambler- y se caracterizan por ser capaces de hidrolizar cefalosporinas de tercera generación y monobactámicos, a diferencia de las betalactamasas de amplio espectro o clásicas pertenecientes al grupo $2 b$-de quienes derivan- que solo actúan frente a penicilinas y cefalosporinas de bajo espectro (8). Las BLEE no hidrolizan carbapenémicos y son sensibles frente a los inhibidores de betalactamasas como el ácido clavulánico (9).

La proliferación de cepas productoras de BLEE constituye un problema epidemiológico considerable, puesto que limita las opciones de tratamientos al ser resistentes a una amplia gama de antimicrobianos tales como las cefalosporinas de segunda, tercera generación y monobactámicos, esto conlleva a fracasos terapéuticos, estancias hospitalarias prolongadas $\mathrm{y}$, por consiguiente, al aumento en los costos de los tratamientos (10).

A pesar de que han sido numerosos los estudios dedicados a este tema, poco han sido los realizados en la región del Magdalena, 
específicamente en la ciudad de Santa Marta. Adicional a esto, es relevante mencionar que la prevalencia de cepas productoras de BLEE varía entre regiones geográficas, por lo cual son más útiles los datos a nivel local o regional para generar pautas sobre los tratamientos de infecciones causadas por estas cepas $(11,12)$. Teniendo en cuenta lo anterior, el objetivo del presente estudio consiste en detectar por medio de pruebas fenotípicas la presencia de cepas de K. pneumoniae productoras de BLEE en un centro clínico de Santa Marta, y a su vez determinar el patrón de resistencia general de dichas cepas.

\section{MATERIALES Y MÉTODOS}

\section{Aislamientos de cepas bacterianas}

Las muestras fueron colectadas durante el periodo comprendido entre los meses de septiembre de 2014 a marzo de 2015. Se aislaron un total de 60 cepas de K. pneumoniae provenientes de cultivos de diferentes muestras biológicas (orinas, sangre, secreciones, líquidos corporales y heces) de pacientes de ambos sexos en un centro clínico de alta complejidad en la ciudad de Santa Marta. Su identificación se llevó a cabo mediante el sistema de identificación de Enterobacteriaceae API ${ }^{\circledR} 10$ S (Biomerieux SA, MarcyI'Etoile, France).

\section{Pruebas de sensibilidad}

Las pruebas de sensibilidad de las cepas bacterianas se realizaron mediante la implementación de antibiogramas para enterobacterias ATB ${ }^{\mathrm{TM}}$ G-5 (Biomerieux SA, MarcyI'Etoile, France). Esta galería consta de 16 cúpulas con una o dos concentraciones de antibióticos, las dos primeras de control. Los antimicrobianos contenidos en dicha galería son: AMO (amoxicilina [8-16 mg/L] ), AMC (amoxilicina + ácido clavulánico [8/4 mg/L] [16/8 mg/L]), PIC (piperacilina [16 mg/L]), TZP (piperacilina + tazobactam [16/4 mg/L]), TIC (ticarcilina [16 mg/L]), TCC (ticarcilina + ácido clavulánico [16/2 mg/L]), CFT(cefalotina [8 mg/L]), CXT (cefoxitine [8]), CTX (cefotaxime [8 mg/L] [32 mg/L]), CAZ (ceftazidime [8 $\mathrm{mg} / \mathrm{L}]$ [16 mg/L]), FEP (cefepime [8 mg/L] [16 $\mathrm{mg} / \mathrm{L}]$ ), CXM (cefuroxime [8 mg/L]), MERO (meropenem [4 mg/L]), IMI (imipenem [4 mg/L] [8 mg/L]), TSU (trimetoprim/sulfametoxazol [2/38 mg/L]), TOB (tobramicina [4 mg/L]), AKN (amikacina [16 mg/L]), GEN (gentamicina [4 mg/L]), NET (netilmicina [8 mg/L]) y CIP (ciprofloxacino [1 mg/L] [2 mg/L]). Para aquellas muestra de origen urinario se utilizó la galería $\mathrm{ATB}^{\mathrm{TM}} \mathrm{UR} 5$ que difiere por la ausencia de TZP, TIC, TCC, MERO, FEP, en su reemplazo tiene NAL (ácido nalidíxico [16 mg/L]), FOS (fosfomicina [64 mg/L]), FUR (nitrofurantoina [32 mg/L]) y NOR (norfloxacino [4 mg/L] [8 $\mathrm{mg} / \mathrm{L}])$ que son antibióticos específicos para vías urinarias.

La lectura de estos antibiogramas permite clasificar las cepas frente a la actividad in vitro de cada uno de los antibióticos, como sensibles $(S)$, intermedias (I) o resistentes (R) mediante la lectura visual de cada cúpula, donde la presencia de turbidez indica resistencia a dicho antibiótico según indicaciones del fabricante. Se catalogaron como sospechosas aquellas cepas que presentaron sensibilidad intermedia o resistencia a CTX y CAZ para aplicarles la prueba fenotípica confirmatoria.

\section{Pruebas confirmatorias}

Esta prueba se realizó por medio del método de difusión con doble disco como se describe en el CLSI (13), para tal fin se contó con placas que contenían el medio de cultivo Agar MuellerHinton inoculado con la suspensión bacteriana de K. pneumoniae dentro del cual se ubicaron cuatro sensidiscos de antibióticos, CAZ $(30 \mu \mathrm{g}) \mathrm{y}$ CTX (30 $\mu \mathrm{g})$ solos y en combinación con el ácido clavulánico, CAZ-CLA (30-10 $\mu \mathrm{g})$ y CTX-CLA (30-10 $\mu \mathrm{g})$. Manteniéndose a una distancia entre sí de 2,5 a $3 \mathrm{~cm}$, posteriormente se incubaron a una temperatura de $35 \pm 2^{\circ} \mathrm{C}$ por 24 horas. 
Se consideró a las cepas como K. pneumoniae productoras de BLEE (K. pneumoniae-BLEE) cuando presentaron un aumento $\geq 5 \mathrm{~mm}$ en el halo de inhibición del disco con ácido clavulánico con respecto a las que contenían el antibiótico solo (13).

\section{RESULTADOS}

Se aislaron en total 652 muestras bacteriológicas, de las cuales 60 correspondieron a la especie K. pneumoniae. De las 60 cepas, 23 fueron consideradas sospechosas de ser productoras de BLEE, pero solo a 21 muestras se les pudo aplicar las pruebas fenotípicas confirmatorias, puesto que 2 de ellas se contaminaron en el proceso. Del total de cepas de K. pneumoniae, 18 (30\%) fueron productoras de BLEE, distribuidas de la siguiente manera: 9/18 (50\%) a partir de hemocultivos, 4/18 (22\%) de secreciones, 3/18 (17\%) de orina y un $2 / 18$ (11\%) de coprocultivos.

Las K. pneumoniae-BLEE se caracterizaron por presentar resistencia en general a las cefalosporinas de primera y segunda generación utilizadas, con la excepción de lacefoxitina donde el $78 \%$ de las cepas fue sensible como se muestra en la Tabla 1. En relación a las cefalosporinas de tercera generación un $94 \%$ fue resistente a cefotaxime, mientras que un $78 \%$ lo fue frente a ceftazidime. Frente a la acción del cefepime un 53,3\% tuvo sensibilidad intermedia, $40 \%$ resistente y solo un $6,7 \%$ fue sensible. Meropenem e imipenem, por su parte, presentaron los mayores porcentajes de sensibilidad con un $80 \%$ y $83 \%$ respectivamente.

El comportamiento de estas cepas fue variado frente al grupo de los aminoglucósidos, presentando mayores valores de resistencia frente a la tobramicina (94\%), seguido de la gentamicina $(83 \%)$, la netilmicina (56\%) y por último la amikacina, que junto con la fosfomicina (en el caso de muestras urinarias) fueron los antibióticos a los cuales no se presentó ningún tipo de resistencia. Con respecto a la ciprofloxacina, el $56 \%$ de las cepas fue sensible frente a la acción de este antimicrobiano. Para el caso de las muestras de origen urinario, se observó un $66,7 \%$ de resistencia al ácido nalidíxico y norfloxacino, iguales porcentajes de sensibilidad y resistencia para la nitrofurantoina (33,3\%), y por ultimo un $100 \%$ de sensibilidad a la fosfomicina como se había mencionado anteriormente.

En la Tabla 2 se muestra el patrón de resistencia general exhibido por las cepas productoras de BLEE. 
Detección de cepas de Klebsiella pneumoniae productoras de betalactamasas de espectro extendido...

Tabla 1. Perfiles de susceptibilidad de las cepas de K. pneumoniae-BLEE.

\begin{tabular}{cccc}
\hline & RESISTENTE & $\begin{array}{c}\text { SENSIBILIDAD } \\
\text { INTERMEDIA }\end{array}$ & SENSIBLE \\
\hline ANTIBIÓTICOS & & & \\
AMO & $100 \%(18 / 18)$ & $0 \%(0 / 18)$ & $0 \%(0 / 18)$ \\
AMC & $72 \%(13 / 18)$ & $17 \%(3 / 18)$ & $11 \%(2 / 18)$ \\
PIC & $100 \%(18 / 18)$ & $0 \%(0 / 18)$ & $0 \%(0 / 18)$ \\
TZP & $80 \%(12 / 15)$ & $6,7 \%(1 / 15)$ & $13,3 \%(2 / 15)$ \\
TIC & $100 \%(15 / 15)$ & $0 \%(0 / 15)$ & $0 \%(0 / 15)$ \\
TCC & $93,3 \%(14 / 15)$ & $6,7 \%(1 / 15)$ & $0 \%(0 / 15)$ \\
CFT & $100 \%(18 / 18)$ & $0 \%(0 / 18)$ & $0 \%(0 / 18)$ \\
CXT & $17 \%(3 / 18)$ & $6 \%(1 / 18)$ & $78 \%(14 / 18)$ \\
CXM & $100 \%(18 / 18)$ & $0 \%(0 / 18)$ & $0 \%(0 / 18)$ \\
CTX & $94 \%(17 / 18)$ & $6 \%(1 / 18)$ & $0 \%(0 / 18)$ \\
CAZ & $78 \%(14 / 18)$ & $6 \%(1 / 18)$ & $17 \%(3 / 18)$ \\
FEP & $40 \%(6 / 15)$ & $53,3 \%(8 / 15)$ & $6,7 \%(1 / 15)$ \\
MERO & $13,3 \%(2 / 15)$ & $6,7 \%(1 / 15)$ & $80 \%(12 / 15)$ \\
IMI & $11 \%(2 / 18)$ & $6 \%(1 / 18)$ & $83 \%(15 / 18)$ \\
TSU & $33 \%(6 / 18)$ & $6 \%(1 / 18)$ & $61 \%(11 / 18)$ \\
TOB & $94 \%(17 / 18)$ & $6 \%(1 / 18)$ & $0 \%(0 / 18)$ \\
AKN & $0 \%(0 / 18)$ & $22 \%(4 / 18)$ & $78 \%(14 / 18)$ \\
GEN & $83 \%(15 / 18)$ & $0 \%(0 / 18)$ & $17 \%(3 / 18)$ \\
NET & $56 \%(10 / 18)$ & $22 \%(4 / 18)$ & $22 \%(4 / 18)$ \\
CIP & $11 \%(2 / 18)$ & $33 \%(6 / 18)$ & $56 \%(0 / 18)$ \\
NAL & $66,7 \%(2 / 3)$ & $0 \%(0 / 3)$ & $33,3 \%(1 / 3)$ \\
FUR & $33,3 \%(1 / 3)$ & $33,3 \%(1 / 3)$ & $100 \%(3 / 3)$ \\
NOR & $66,7 \%(2 / 3)$ & $0 \%(0 / 3)$ & \\
FOS & $0 \%(0 / 3)$ & $0 \%(0 / 3)$ & $(1 / 3)$ \\
\hline & & & \\
\hline
\end{tabular}

AMO: amoxicilina, AMC: amoxicilina/ácido clavulánico, PIC: piperacilina, TZP: piperacilina/tazobactam, TIC: ticarcilina, TCC: ticarcilina/ácido clavulánico, CFT: cefalotina, CXT: cefoxitine, CXM: cefuroxime, CTX: cefotaxime, CAZ: ceftazidime, FEP: cefepime, MERO: meropenem, IMI: imipemems, TSU: trimetoprim/sulfametoxazol, TOB: tobramicina, AKN: amikacina, GEN: gentamicina, NET: netilmicina, CIP: ciprofloxacino. (El espacio sombreado pertenece a muestra de origen urinario).

Tabla 2. Patrón de resistencia fenotípica de las cepas de K. pneumoniae-BLEE. R: resistente, S: sensible, I: intermedio.

\begin{tabular}{ccccccccccccccccccccccccccc}
\hline AMO & AMC & PIC & TZP & TIC & TCC & CFT & CXT & CTX & CAZ & FEP & CXM & MERO & IMI & TSU & TOB & AKN & GEN & NET & CIP & NAL & FUR & NOR & FOS \\
\hline $\mathbf{R}$ & $\mathbf{R}$ & $\mathbf{R}$ & $\mathbf{R}$ & $\mathbf{R}$ & $\mathbf{R}$ & $\mathbf{R}$ & $\mathbf{s}$ & $\mathbf{R}$ & $\mathbf{R}$ & $\mathbf{l} / \mathbf{R}$ & $\mathbf{R}$ & $\mathbf{s}$ & $\mathbf{s}$ & $\mathbf{s}$ & $\mathbf{R}$ & $\mathbf{s}$ & $\mathbf{R}$ & $\mathbf{R}$ & $\mathbf{s} / \mathbf{I}$ & $\mathbf{R}$ & $\mathbf{1} / \mathbf{R}$ & $\mathbf{R}$ & $\mathbf{s}$ \\
\hline
\end{tabular}




\section{DISCUSIÓN}

Las BLEE constituyen un problema epidemiológico creciente, que involucra no solo al campo de la medicina y farmacología, sino que también afecta la economía de las entidades de salud (10). Estas enzimas son una de las causantes de graves infecciones nosocomiales, debido a que las cepas que las producen además de ser resistentes frente a la acción de cefalosporinas de tercera generación y monobactámicos, lo son también frente a otros grupos de antibióticos, debido a que usualmente los genes que codifican las BLEE son transferidos de una bacteria a otra en conjunto con otros genes que codifican resistencia a otro tipos de antimicrobianos (8). Por consiguiente, las cepas productoras de BLEE se han convertido en un problema de salud a nivel mundial (12).

En este contexto, encontramos que la prevalencia de K. pneumoniae-BLEE en América Latina es de $45,4 \%$, seguido de la región Pacífica occidental (24,6\%), Europa (22,6\%), Estados Unidos $(7,6 \%)$ y Canadá (4,9\%) (11). Dentro de Latinoamérica encontramos las tasas de prevalencias más altas en países como Honduras, Chile, Guatemala y Brasil cuyos porcentajes superan el 45\%, situándose Colombia y Venezuela en los últimos lugares con porcentajes del 18,7\% y 17,2\% respectivamente según datos del programa T.E.S.T. 2004-2010 (14).

En particular para Colombia, Sánchez et al. (15) reportaron porcentajes del 9,52\% de aislamientos de K. pneumoniae-BLEE en Villavicencio; valores bajos con respecto a lo reportado en hospitales de Medellín (16), Montería (17) y Bogotá (18), donde las tasas de prevalencia fueron del $33 \%$, $46 \%$ y $43,5 \%$ respectivamente. Siendo similares los resultados aquí obtenidos con un 30\% de cepas productoras.

En relación a los patrones de resistencia observados en este estudio, se obtuvo que para las combinaciones de antibióticos con inhibidores de betalactamasas los porcentajes fueron altos, a pesar de que por definición las
BLEE son inhibibles por estas sustancias (9). Esto no necesariamente ha de ser consecuencia de la producción de estas enzimas, sino que puede deberse a la expresión de varios mecanismos de resistencia coexistentes (19).

Los carbapenems, por su parte, fueron los antibióticos con valores de sensibilidad más altos, sin embargo, se encontraron pequeños porcentajes de resistencia a estos antimicrobianos (13,3\% para meropenem y $11 \%$ para imipenem), lo cual contrasta con los resultados reportados en trabajos como los de Sánchez et al. (15), Morales et al. (20) y Torres et al. (21) donde no se obtuvo ninguna resistencia. Aunque poca, esta disminución de la sensibilidad puede deberse ya sea a varios mecanismos de resistencia actuando conjuntamente, tales como la producción de BLEE y modificación de la permeabilidad en la membrana (22), o a la diseminación de cepas productoras de carbapenemasas, teniendo en cuenta que Colombia es el primer país de Latinoamérica donde se reportaron estas enzimas (23), afirmaciones para lo cual se necesitarían estudios posteriores.

Es de resaltar que la amikacina, pese a tener valores de sensibilidad un poco más bajos que los carbapenems, fue el único antimicrobiano al cual ninguna cepa fue resistente. Son varios los estudios que apoyan la buena actividad de este antimicrobiano $(14,24,25)$.

En cuanto a la fosfomicina vale destacar que, aunque es citado en algunos estudios como un fármaco con limitada actividad frente a $K$. pneumoniae-BLEE (26), en el presente trabajo fue el antibiótico con mejor actividad in vitro frente a las cepas de origen urinario, obteniendo un $100 \%$ de sensibilidad. Trabajos como los de De Cueto et al. (27) apoyan estos resultados reportando un $92,8 \%$ de sus cepas como sensibles frente a la acción de este antibiótico.

En conjunto las K. pneumoniae-BLEE asiladas en este estudio exhibieron un patrón fenotípico caracterizado por la resistencia 
a las aminopenicilinas, ureidopenicilinas, carboxipenicilinas y cefalosporinas de primera, segunda y tercera generación como lo son estas cepas por definición. Presentaron, también, resistencia a las combinaciones con inhibidores de bectalactamasas, así como a algunos aminoglucósidos tales como tobramicina, gentamicina y netilmicina. Manteniendo la sensibilidad a la amikacina, trimetoprim/ sulfametoxazol, quinolonas y carbapenems.

\section{REFERENCIAS}

1. Navarro F, Miro E, Mirelis B. Lectura interpretada del antibiograma de enterobacterias. Enferm Infecc Microbiol Clin 2010; 28(9):638-645.

2. Ambler RP. The structure of $\beta$-lactamases. Philos Trans R Soc Lond B Biol Sci 1980; 289:321-331.

3. Wilke MS, Lovering AL, Strynadka CJ. $\beta$-Lactam antibiotic resistance: a current structural perspective. Curr Opin Microbiol 2005; 8:525-533.

4. Almanza D. Identificación de betalactamasas de espectro extendido en enterobacterias. Revista Habanera de Ciencias Médicas 2010; 9(4):516-524.

5. Livermore D. b-Lactamases in Laboratory and Clinical Resistance. Clinical microbiology reviews 1995; 8(4):557-584.

6. Pérez F, Endimiani A, Hujer K, Bonomo R. The continuing challenge of ESBLS. Curr Opin Pharmacol 2007; 7(5):459-69.

7. Bush K, Jacoby GA, Medeiros A. A functional classification scheme forbeta-lactamases and its correlation with molecular structure. Antimicrob Agents Chemother 1995; 39(6):1211-1233.

8. Pujol M, Peña C. El significado clínico de las betalactamasas de espectro extendido. Enferm Infecc Microbiol Clin 2003; 21(2):69-71.

9. Seral C, Pardos M, Castillo F. Betalactamasas de espectro extendido en enterobacterias distintas de Escherichia coli y Klebsiella pneumoniae. Enferm Infecc Microbiol Clin 2010; 28(1):12-18.

10. Schwaber M, Venezia S, Kaye K, Ben-Ami R, Schwartz D, Carmeli Y. Clinical and Economic Impact of Bacteremia with Extended Spectrum-Lactamase-Producing Enterobacteriaceae. Antimicrobial agents and chemotherapy 2006; 50(4):1257-1262.

11. Winokur PL, Canton R, Casellas JM, Legakis N. Variations in the Prevalence of Strains Expressing an Extended-Spectrum $\beta$-Lactamase Phenotype and Characterization of Isolates from Europe, the Americas, and the Western Pacific Region. Clinical Infectious Diseases 2001; 32(2):94-103.

12. Pfaller M, Segreti J. Overview of the Epidemiological Profile and Laboratory Detection of ExtendedSpectrum b-Lactamases. Clin Res Infect Dis 2006; 42(4):153-163.

13. CLSI. Performance standards for antimicrobial susceptibility testing; twenty second informational supplement. Eleventh edition. Wayne, Pennsylvania: Clinical and Laboratory Standards Institute; 2012.

14. Fernández-Canigia L, Dowzicky M. Susceptibility of important Gram-negative pathogens to tigecycline and other antibiotics in Latin America between 2004 and 2010. Annals of Clinical Microbiology and Antimicrobials 2012; 11:29-37.

15. Sánchez L, Ríos R, Mattar S. Detección de beta-lactamasas de espectro extendido en Escherichia coli y Klebsiella pneumoniae aislados en una clínica de Villavicencio, Colombia. Asociación Colombiana de Infectología 2008; 12(3):193-200.

16. Echeverri-Toro LM, Rueda Z, Maya W, Agudelo Y, Ospina S. Klebsiella pneumoniae multi-resistente, factores predisponentes y mortalidad asociada en un hospital universitario en Colombia. Rev Chil Infect $2012 ; 29(2): 175-182$. 
17. Martínez $P$, Mercado $M$, Mattar $S$. Determinación de $\beta$-Lactamasas de espectro extendido en gérmenes nosocomiales del hospital San Jerónimo, Montería. Acta Med Colomb 2003; 34(4):196-205.

18. Garzón J, Lemos E, Rivas R. Prevalencia de betalactamasas de espectro extendido en Escherichia coli, Klebsiella pneumoniae y Klebsiella oxytoca del Hospital Occidente de Kennedy. Nivel III, Bogotá. Revista ciencias de la salud 2004; 2(2):124-38.

19. Paterson DL. Tratamiento de las infecciones por microorganismos productores de BLEE. Enferm Infecc Microbiol Clin 2007; 25(2):60-63.

20. Morales JL, Reyes K, Monteghirfo M, Roque M, Irey J. Presencia de $\beta$-lactamasas de espectro extendido en dos hospitales de Lima, Perú. An Fac Med Lima 2005; 66(1):24-32.

21. Torres L, Gagliotta V, Torres O, Benítez M, Domínguez M, Pedroza R. $\beta$-Lactamasas de Espectro Expandido en Enterobacterias aisladas en Centros de Salud de Caracas. Sociedad Venezolana de Microbiología 2006; 26:80-88.

22. Jacoby G, Mills D, Chow N. Role of B-Lactamases and Porins in Resistance to Ertapenem and Other B-Lactams in Klebsiella pneumoniae. Antimicrob Agents Chemother 2004; 48(8):3203-3206.

23. Villegas M, Lolans K, Correa A, Suárez C, López J, Vallejo M, Quinn J. First Detection of the PlasmidMediated Class A Carbapenemase KPC-2 in Clinical Isolates of Klebsiella pneumoniae from South America. Antimicrob Agents Chemother 2006; 50(8):2880-2882.

24. Díaz P, Bello H, Domínguez M, Trabal N, Mella S, Zemelman R, González G. Resistencia a gentamicina, amikacina y ciprofloxacina en cepas hospitalarias de Klebsiella pneumoniae subespecie pneumoniae productoras de B-lactamasas de espectro extendido. Rev Méd Chile 2004; 132:1173-1178.

25. Gaitán S, Espinal P, Grupo de Investigación en Resistencia Bacteriana, Región Caribe. Caracterización molecular de Escherichia coli y Klebsiella pneumoniae productores de $\beta$-lactamasas de espectro extendido en hospitales de la Región Caribe, Colombia. Rev Chil Infect 2009; 26(3):239-246.

26. Hernández MS, García JA, Muñoz JL. Actividad in vitro de fosfomicina frente a enterobacterias de origen urinario productoras de betalactamasas de espectro extendido. Rev Esp Quimioter 2009; 22(1):25-29.

27. De Cueto M, Hernández J, López-Cerero L, Morillo C, Pascual A. Actividad de fosfomicina sobre cepas de Escherichia coli y Klebsiella pneumoniae productoras de betalactamasas de espectro extendido. Enferm Infecc Microbiol Clin 2006; 24(10):613-616. 